\title{
Síndrome moyamoya en paciente con síndrome de Down y déficit de antitrombina III
}

\author{
Marta Hernández $\mathrm{Ch}^{1}$, Isidro Huete $\mathrm{L}^{2}$, \\ María-José Concha G 3 , José I Méndez $C^{3 a}$, \\ Nicolás Sánchez $D^{3}$, María-Carolina Cuéllar $\mathbf{G}^{33}$, \\ Eva Retamal $\mathbf{R}^{3 a}$, Tomás Mesa $\mathrm{L}^{\mathbf{1}}$.

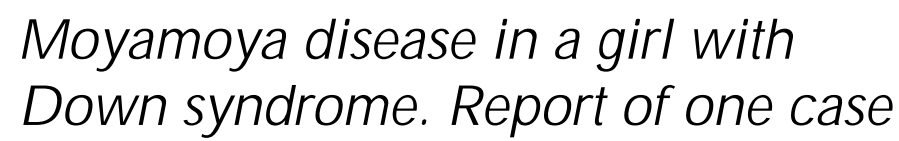

Moyamoya disease is a unique chronic progressive cerebrovascular disease characterized by bilateral stenosis or occlusion of the arteries around the circle of Willis with prominent arterial collateral circulation. It can be primary or secondary to genetic syndromes such as Down syndrome. We report a seven year-old girl with a Down syndrome that presented with a disturbance of consciousness, seizures and a right hemiparesia at the age of five. Magnetic resonance imaging showed old cortical ischemic lesions in both cerebral hemispheres and a recent infarction in the territory of the left middle cerebral artery. Brain angiography showed a proximal stenosis of both medial cerebral arteries and a net of collateral vessels, consistent with the diagnosis of moyamoya syndrome. The patient had also an antithrombin III deficiency. Aspirin was indicated and a surgical correction was recommended. However, prior to the procedure, the patient had a new infarction in the territory of the right middle cerebral artery, which caused a severe disability (Rev Méd Chile 2009; 137: 1066-70).

(Key words: Antithrombin III deficiency; Cerebrovascular Moyamoya disease; Down syndrome)

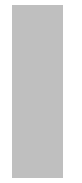

\footnotetext{
Recibido el 6 de agosto, 2008. Aceptado el 30 de abril, 2009.

${ }^{1}$ Departamento de Pediatría, Sección Neurología Infantil, ${ }^{2}$ Departamento de Radiología, ${ }^{3}$ Escuela de Medicina, Facultad de Medicina, Pontificia Universidad Católica de Chile. Santiago de Chile.

${ }^{a}$ Internos Escuela de Medicina
}

$\mathrm{L}$

a enfermedad moyamoya (EMM) fue descrita por primera vez en Japón en 1957, por Takeuchi y Shimizu ${ }^{1}$. Es un trastorno cerebrovascular oclusivo crónico, de origen desconocido,

Correspondencia a: Dra. Marta Hernández Ch. Departamento de Pediatría, Facultad de Medicina, Pontificia Universidad Católica de Chile. Lira 85, Santiago, Chile. C.P. 6510273. Tel: 3546486. Fax: 6384307. E mail: mhernand@med.puc.cl caracterizado por la obstrucción progresiva de las grandes arterias del polígono de Willis como la carótida interna en su porción supraclinoidea y segmentos proximales de las cerebrales anteriores o medias. La base anatomopatológica consiste en una proliferación de las células musculares lisas y su migración hacia la íntima por mecanismos desconocidos, lo que origina una estenosis arterial. El hallazgo angiográfico típico, además de la oclusión de las carótidas, es el desarrollo de una frágil red basal de vasos colaterales denominados 
"moyamoya" que en japonés significa "volutas de humo" y que define esta vasculopatía ${ }^{2}$. La EMM ocurre preferentemente en asiáticos, con una incidencia de 50,7 por 100.000 habitantes $^{3}$. El síndrome moyamoya (SMM) tiene un patrón angiográfico similar, pero la vasculopatía subyacente es secundaria a enfermedades autoinmunes, tu-

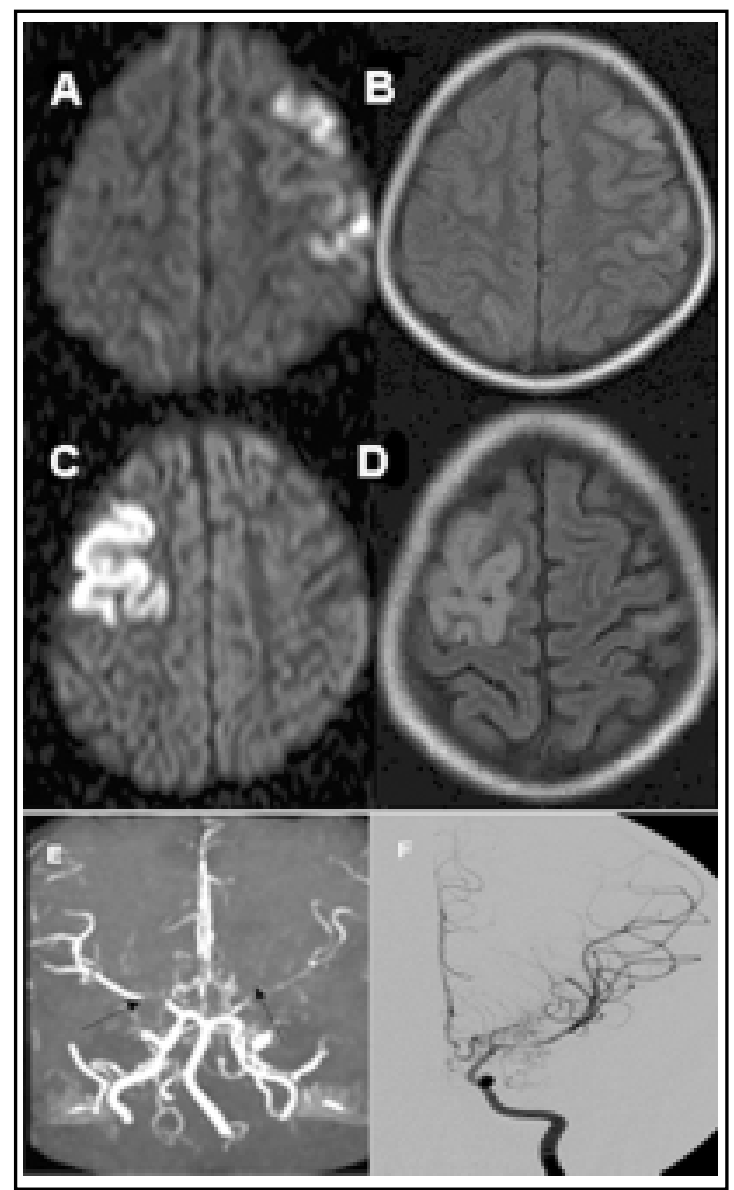

Figura 1. Secuencias Diw $(A, C)$ y FLAIR $(B, D)$. A y B (primer infarto): lesiones hiperintensas corticales frontales izquierdas y una más pequeña parietal derecha, con restricción de la difusión. C y D (segundo infarto): lesiones isquémicas similares en territorio carotídeo derecho. E: AngioRM cerebral. Se observan lesiones estenosantes en segmentos M1 con mayor compromiso izquierdo. F: Angiografía de carótida interna izquierda. Confirma estenosis en arteria cerebral media izquierda y evidencia el desarrollo de vasos penetrantes basales. mores cerebrales, irradiación, síndrome de Down (SD), neurofibromatosis tipo 1, meningitis bacteriana, tuberculosis, poliarteritis nodosa, anemia de células falciformes y tetralogía de Fallot, entre otros ${ }^{4}$.

Los pacientes con SD desarrollan SMM con mayor frecuencia que la población general y éste se presenta con un amplio rango de síntomas neurológicos, como compromiso de conciencia recurrente, convulsiones, déficit motor o sensorial, movimientos involuntarios, cefalea o deterioro cognitivo ${ }^{5}$. Pearson et al en un análisis de ACV en 27 niños con SD encontró 7 pacientes con alteraciones angiográficas compatibles con un $\mathrm{SMM}^{6}$. Para establecer el diagnóstico, es necesario un alto grado de sospecha y confirmación con angioRM o angiografía convencional.

\section{Caso clínico}

Presentamos el caso de una niña de 7 años con SD, sin cardiopatía congénita ni antecedentes perinatales relevantes. Portadora de cariotipo $47 X X+21$, hipotiroidismo y laringomalacia. Su madre no refirió antecedentes de cefaleas ni epilepsia. Acudía regularmente a escuela de educación diferencial y era autovalente en actividades de la vida diaria a los 5 años, ingresó a la UCI pediátrica por compromiso cualicuantitativo de conciencia, afasia, hemiparesia facio-braquio-crural y posterior convulsión autolimitada de hemicuerpo derecho. Al examen físico destacaban dismorfias características de SD, sopor superficial con respuesta a estímulos verbales y afasia. Al examen neurológico presentaba fondo de ojo normal, asimetría facial evidente de origen central y hemiparesia derecha. El examen cardiovascular era normal, sin soplos carotídeos y pulsos periféricos normales. La ecografía cardiaca resultó normal. La tomografía computada (TC) cerebral informó infarto reciente en corona radiada y corteza frontal izquierda, lo que fue corroborado por la RM, que además mostró infartos antiguos supratentoriales bilaterales múltiples que comprometían centros semiovales en territorios limítrofes y corteza frontoparietal izquierda (Figura 1). La angiografía por RM mostró lesiones estenosantes e irregulares en los segmentos M1 y alteraciones en la pared de los segmentos P1 y P2, donde había 
estenosis y dilataciones irregulares bilaterales. En los sifones carotídeos se observó calibre irregular y estenosis distal. Por último, la angiografía por sustracción digital evidenció la presencia de arteriopatía cerebral tipo moyamoya, mayor al lado izquierdo.

El tamizaje para enfermedades autoinmunes fue negativo. El estudio de trombofilias mostró una función de antitrombina III bajo lo normal (73\%). El valor a los seis meses se mantuvo alterado (67\%), considerando el rango normal ajustado por edad entre $76 \%$ y $113 \%$.

La paciente evolucionó favorablemente, recuperando el déficit motor y se dio de alta con terapia antiagregante plaquetaria (aspirina $3 \mathrm{mg} /$ $\mathrm{kg} /$ día) previa a cirugía para revascularización quirúrgica. Al mes de control sólo presentaba leve paresia facial central, con marcha normal y estado mental similar al basal. Se reintegró adecuadamente a su escuela.

En el control con neuroimágenes a los 3 meses evidenció un aumento de la estenosis arterial y aparición de nuevos microinfartos silentes.

En espera de cirugía presentó sepsis severa de foco respiratorio, por lo que fue hospitalizada nuevamente. En este contexto, presentó una crisis convulsiva de hemicuerpo izquierdo, con neuroimágenes que informaron nuevo infarto en territorio correspondiente a rama anterior de ACM derecha. Durante esta hospitalización la paciente presentó mala evolución, requiriendo drogas vasoactivas por inestabilidad hemodinámica. Fue gastrostomizada y presentó múltiples complicaciones postoperatorias. Además cursó con presiones arteriales (PA) elevadas, de difícil manejo. Dentro del estudio, se realizó angioTC renal, que no mostró hallazgos patológicos. El estudio con Holter mostró correlación de elevación de PA con períodos de estrés. La RM de cerebro, previa al egreso, mostró secuelas extensas de infartos con grandes zonas de atrofia cortical y de gliosis. Se dio de alta en estado de mínima conciencia, y con régimen de hospitalización domiciliaria.

\section{DisCUSIÓN}

Los niños con SD tienen un riesgo mayor de ACV isquémico, generalmente secundario a tromboem- bolismo cardíaco e infecciones bacterianas incluyendo meningitis y endocarditis ${ }^{6}$. El SMM es una causa poco común de ACV en pacientes con SD, pero su incidencia es 3 veces mayor que la incidencia esperada para la población general ${ }^{7,8}$.

La edad de inicio de los síntomas del SMM tiene una distribución bimodal, con un alza en la primera y cuarta década de la vida y con distinta presentación según la edad. En niños suele haber signos de isquemia cerebral y en adultos se presenta principalmente con hemorragias. En niños con síndrome de Down la estenosis ocurre más precozmente y es más agresiva que en la población general ${ }^{9,10}$. Nuestro paciente se presentó con múltiples ACV isquémicos entre los 5 y 7 años de edad.

Los vasos moyamoya se desarrollan en un lapso de 1 a 2 años. Durante este período, puede haber síntomas como crisis isquémicas transitorias (CIT) recurrentes, parálisis sensorio-motoras, convulsiones o cefaleas tipo migraña ${ }^{11}$. Nuestro paciente presentó en 2 oportunidades crisis de "palidez y fatiga", autolimitadas, que motivaron consulta médica, atribuyéndose a infecciones respiratorias.

La edad promedio de diagnóstico de SMM en niños con SD es de 9,5 años, dos años más tarde que en los niños sin esta patología (7,1 años). Esta variación probablemente se debe a una falta de evaluación acuciosa de síntomas como convulsiones o eventos paroxísticos cerebrales en la población de niños con SD.

En nuestra paciente se hizo el diagnóstico a los 5 años, pero los antecedentes descritos nos hacen pensar que había presentado CIT previas, atribuidas a cuadros respiratorios virales. Esta sospecha es apoyada por los infartos antiguos descritos en la RM.

Hay varias hipótesis para la asociación de SD y SMM, entre ellos, la morfología anormal del lecho capilar, la alta resistencia vascular pulmonar, la asociación con cardiopatía congénita, la anomalía de vasos retinianos y fibrodisplasia primaria de la íntima ${ }^{12}$. En relación a la base molecular del SD, varias proteínas del cromosoma 21, como cadenas $\alpha$ de colágeno tipo VI, súper óxido dismutasa I, receptor de interferón gama y cistationina $\beta$ $\operatorname{sintasa}^{13}$, están asociadas a mayor riesgo de enfermedad cardiovascular. La autoinmunidad es otro factor posible, ya que los pacientes con SD tienen 
mayor prevalencia de trastornos autoinmunes como tiroiditis ${ }^{14}$. La subluxación cervical superior también puede llevar a una insuficiencia circulatoria y predisponer al desarrollo de $\mathrm{SMM}^{15}$. En nuestro paciente no había evidencia de subluxación cervical ni enfermedades autoinmunes. Tampoco presentaba cardiopatía.

No hay casos reportados de SMM, SD y déficit de antitrombina III. Encontramos un caso de SMM más SD asociado a déficit de proteína $\mathrm{C}^{16}$, en el cual atribuían la causa del SMM a este déficit. Creemos que el déficit de antitrombina III es un factor de riesgo en nuestra paciente que se suma al SMM en la patogénesis de ACV isquémico.

Considerando la naturaleza progresiva y tratable de la enfermedad y sus consecuencias potencialmente letales, se recomienda el estudio con RM y angioRM cerebral en pacientes con SD que presenten eventos paroxísticos cerebrales o deterioro intelectual y una vez certificado el diagnóstico, el tratamiento quirúrgico debe implementarse lo antes posible, usando técnicas de bypass extraintracraneano o bien sinagiosis pial ${ }^{17}$.

\section{REFERENCIAS}

1. Takeuchi K, Shimizu K. Hypoplasia of the bilateral internal carotid arteries. Brain Nerve 1957; 9: 37-43.

2. Suzuki J, TAKAKU A. Cerebrovascular "moyamoya" disease: a disease showing abnormal net-like vessels in base of brain. Arch Neurol 1969; 20: 288-99.

3. Ikeda K, Kashihara H, Hosozawa K. Adult moyamoya disease on Japanease brain checkup. J Clin Neurosci 2006; 13: 334-8.

4. Fryer R, Anderson R, Chiriboga C, Feldstein N. Sickle cell anemia with moyamoya disease: outcome after EDAS procedure. Pediatr Neurol 2003; 29: 124-30.

5. Fukushima Y, Kondo Y, Kuroki Y. Are Down syndrome patients predisposed to moyamoya disease? Eur J Pediatr 1986; 144: 516-7.

6. Pearson E, Lenn NJ, Cail WS. Moyamoya and other causes of stroke in patients with Down syndrome. Pediatr Neurol 1985; 1: 174-9.

7. Goldstein EM, Singer HS. Moyamoya-like disease in Down's syndrome. Pediatr Neurosurg 1990-1991; 16: $14-6$

8. DAi AI, Shaikh ZA, Cohen ME. Early-onset Moyamoya syndrome in a patient with Down syndrome: Case report and review of the literature. J Child Neurol 2000; 15: 696-9.
En cuanto a tratamiento preventivo, en el niño, a diferencia del adulto, la ausencia de estudios randomizados y las diferencias en cuanto a sistemas de coagulación, neurológico y cerebrovasculares, limitan la aplicabilidad de los tratamientos indicados en el adulto. Las recomendaciones corrientes para el tratamiento del evento agudo y la prevención, están basados en pequeños estudios no randomizados, series de casos, consensos u opiniones de expertos. Las dos guías clínicas publicadas $^{18,19}$ difieren en algunos aspectos, pero logran consenso para la utilización de heparina preventiva para ACV arterial sólo en los casos de anemia drepanocítica y disección arterial. En todas las otras causas de ACV se considera segura y eficaz la terapia preventiva con aspirinas en dosis de $3 \mathrm{mg} / \mathrm{kg} /$ día, que fue lo indicado en nuestra paciente.

Es importante establecer contactos con centros de estudios multicéntricos internacionales ${ }^{20}$, para establecer tratamientos estandarizados en la población infantil que permitan un manejo óptimo y una disminución de la mortalidad y de las secuelas neurológicas permanentes.

9. Fukui M. Current state of study on moyamoya disease in Japan. Surg Neurol 1997; 47: 138-43.

10. Gatenby P, Tucko R, Andrews C, O'Neil E. Antiphospholipid antibodies and stroke in Down syndrome. Lupus 2003; 12: 58-62.

11 Aydin K, Okuyaz C, Gücüyener K, Serdaroglu A, Akpek S. Moyamoya disease presented with migrainelike headache in a 4-year-old girl. J Child Neurol 2003; 18: 361-3.

12. Fleisher GR, Buck BE, Cornfeld D. Primary intimal fibroplasia in a child with Down's syndrome. Am J Dis Child 1978; 132: 700-3. Bull Acad Natl Med 1985; 169: 1387-95.

13. Jerome H, Chadefaux B, Ceballos I, Allard D. Effects of gene localization and its metabolic significance in trisomy 21. Bull Acad Natl Med 1985; 169: 1387-95.

14. Leno C, Mateo i, Cid C, Berciano J, Sedano C. Autoimmunity in Down's Syndrome: Another possible mechanism of moyamoya disease. Stroke 1998; 29: 868-9.

15. Takanashi J, Sugita K, Ishit M, Iai M, Goto M, Tanabe Y, Niım H. A girl with Down syndrome complicated by moyamoya disease and symptomatic atlanto-axial instability. No To Hattatsu 1993; 25: 248-52.

16 Gururaj A, Hardy D, Al-Gazali Li, Sztriha L, Roos A. ¿Are the strokes in moyamoya syndrome associated 
with Down syndrome due to protein $\mathrm{C}$ deficiency? Brain and Development 2002; 24: 719-22.

17. Jea A, Smith ER, Robertson R, Scott RM. Moyamoya syndrome associated with Down syndrome: outcome after surgical revascularization. Pediatrics 2005; 116: e694-701.

18. Monagle P, Chan A, Massicotte P, Chalmers E, MichelSON AD. Antithrombotic therapy in children: the Seventh ACCP Conference on Antithrombotic and
Thrombolytic Therapy. Chest 2004; 126 (3 Suppl): 645-87.

19. Paediatric Stroke Working Group. Stroke in Childhood: Clinical Guidelines for Diagnosis, Management and Rehabilitation. London: Royal College of Physicians of London (UK); 2004.

20. International Paediatric Stroke Study https// app3.ccb.sickkids.ca/cstrokestudy 